\title{
Optimal time-domain combination of the two calibrated output quadratures of GEO 600
}

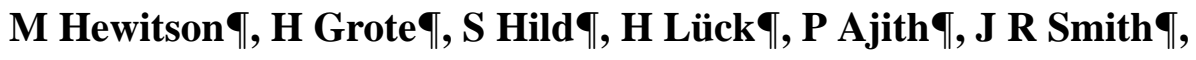 \\ K A Strain $\S$, B Willke $\uparrow$, G Woan $\S$. \\ || Max-Planck-Institut für Gravitationsphysik (Albert-Einstein-Institut) und Universität \\ Hannover, Außenstelle Hannover, Callinstr. 38, 30167 Hannover, Germany. \\ $\S$ Physics \& Astronomy, University of Glasgow, Glasgow G12 8QQ, Great Britain. \\ E-mail: martin.hewitson@aei.mpg • de
}

PACS numbers: 95.55.Br, 95.75.Kk, 04.80.Nn, 95.55.Ym

\begin{abstract}
.
GEO 600 is an interferometric Gravitational Wave detector with a $600 \mathrm{~m}$ armlength and which uses a dual-recycled optical configuration to give enhanced sensitivity over certain frequencies in the detection band. Due to the dual-recycling, GEO 600 has two main output signals, both of which potentially contain Gravitational Wave signal. These two outputs are calibrated to strain using a time-domain method. In order to simplify the analysis of the GEO 600 data set, it is desirable to combine these two calibrated outputs to form a single strain signal that has optimal signal-tonoise ratio across the detection band. This paper describes a time-domain method for doing this combination. The method presented is similar to one developed for optimally combining the outputs of two colocated Gravitational Wave detectors. In the scheme presented in this paper, some simplifications are made to allow its implementation using time-domain methods.
\end{abstract}

\section{Introduction}

GEO 600 [1], is part of an international network of gravitational wave observatories which is searching for gravitational wave signatures from various source types. 
GEO 600 is the only long-baseline dual-recycled Michelson Interferometer in the world. With its $600 \mathrm{~m}$ arm-length, it should, when fully commissioned, be sensitive to strain amplitudes of the order of $1 \times 10^{-22} 1 / \sqrt{\mathrm{Hz}}$.

GEO 600 uses a dual-recycling configuration which means that two additional mirrors are added to the standard Michelson Interferometer optical layout. One mirror, the power-recycling (PR) mirror, is added at the input port of the Michelson. Since the Michelson interferometer in GEO 600 is operated at a dark fringe, the light reflected from the input port is made resonant by the cavity formed between the PR mirror and the Michelson. Another mirror, the signal-recycling (SR) mirror, is placed at the output of the Michelson to create a resonant cavity for any signal sidebands that leave the interferometer (see $[2,3]$ for details).

The use of signal-recycling gives an enhancement in strain sensitivity over a certain band of frequencies. This band is defined by the reflectivity of the SR mirror and the length of the SR cavity. One consequence of using such an optical scheme is that the gravitational wave $(\mathrm{GW})$ signal gained from demodulating the detector output at the frequency of the control sidebands is spread between the two demodulation quadratures. This means that, for a given demodulation phase, the GW signal content in one quadrature can only be optimised for one frequency; at all other frequencies, the demodulation phase is not optimal. In the absence of noise, the complete signal could be recovered by calibrating only one of the output quadratures. However, since there is noise in the system, the data from both output quadratures need to be calibrated such that at any given frequency, the data stream with the best signal-to-noise ratio is available for analysis. Having calibrated both output data streams, we have two estimates for the detected strain of the interferometer. This is somewhat undesirable since a choice must be made, based on the analysis to be performed, as to which strain signal to analyse.

To remove the need for this choice, we seek to combine the two calibrated output signals of GEO 600 into a single strain signal that has an optimal signal-to-noise ratio at all frequencies in the detection band $(40 \mathrm{~Hz}$ to $6 \mathrm{kHz})$. In addition, as we will see, the combination of the two calibrated streams, if done correctly, leads to a signal that is more sensitive than either of the two separate signals. In order to fit in with the current calibration scheme of GEO 600, we want to do this combination in the time-domain. 


\section{Time-domain calibration of GEO 600}

The calibration of GEO 600 is done using a time-domain method. Calibration lines are continuously injected into the differential length-control actuators of the Michelson interferometer at a few frequencies across the detection band. The response of the detector to this differential displacement, and hence to strain, is computed once per second by forming the transfer function from the injection point to the two detector outputs; a model of this transfer function is then fit to the measurements. This model is then inverted and used to create time-domain filters. By filtering the two detector outputs, $P(t)$ and $Q(t)$, through these time-domain filters, we recover two estimates, $h_{\mathrm{P}}(t)$ and $h_{\mathrm{Q}}(t)$, of the apparent detected strain of the interferometer. The details, development, and application of the calibration scheme are given in $[4,5,6,7]$. The method runs in real-time with a latency of a few seconds.

\section{Optimal combination of two calibrated output signals of GEO 600}

The combination of the two calibrated output signals of GEO 600 can be done by considering the variance of the noise in the two signals in a particular frequency bin. Here we wish to combine the two signals together so as to achieve the best estimate of the detected gravitational wave strain. In addition, we want to take into account any correlations that may exist between the two calibrated output signals.

\subsection{Maximum likelihood method of combining two signals}

We start from the assumption that the two calibrated output signals, $h_{\mathrm{P}}$ and $h_{\mathrm{Q}}$, contain the same gravitational wave signals. This is true to a level consistent with the accuracy of the calibration of each output signal. In addition, each calibrated output contains noise components which will, in general, be different, but may be correlated to a varying degree at some frequencies. Then we can write

$$
\begin{aligned}
& h_{\mathrm{P}}(t)=h(t)+N_{\mathrm{P}}(t), \\
& h_{\mathrm{Q}}(t)=h(t)+N_{\mathrm{Q}}(t),
\end{aligned}
$$

where $h(t)$ is the underlying signal we seek, and $N_{\mathrm{P}}(t)$ and $N_{\mathrm{Q}}(t)$ represent the noise in the two calibrated output quadratures. 
The method centres around the covariance matrix of the two signals:

$$
\mathrm{C}=\left(\begin{array}{ll}
\sigma_{\mathrm{PP}} & \sigma_{\mathrm{PQ}} \\
\sigma_{\mathrm{QP}} & \sigma_{\mathrm{QQ}}
\end{array}\right)
$$

where $\sigma_{\mathrm{QP}}=\left\langle\tilde{N}_{\mathrm{P}} \tilde{N}_{\mathrm{Q}}^{*}\right\rangle=\sigma_{\mathrm{PQ}}^{*}$ and ${ }^{\sim}$ denotes Fourier transform. Here, the cross terms $\sigma_{\mathrm{PQ}}$ and $\sigma_{\mathrm{QP}}$ are the variances of those signal components that are common to both $h_{\mathrm{P}}$ and $h_{\mathrm{Q}}$. These terms are, in general, complex functions of frequency.

For a single frequency, $f$, it can be shown that the optimal combination of the two calibrated strain signals is given by

$\hat{h}(f)=\frac{h_{\mathrm{P}}(f) \sigma_{\mathrm{QQ}}(f)+h_{\mathrm{Q}}(f) \sigma_{\mathrm{PP}}(f)-\sigma_{\mathrm{PQ}}(f) h_{\mathrm{P}}(f)-\sigma_{\mathrm{QP}} h_{\mathrm{Q}}(f)}{\sigma_{\mathrm{PP}}(f)+\sigma_{\mathrm{QQ}}(f)-\left[\sigma_{\mathrm{PQ}}(f)+\sigma_{\mathrm{QP}}(f)\right]}$,

where $\hat{h}(f)$ is a maximum likelihood estimate for the underlying gravitational wave strain signature, $h(f)$. An outline of the derivation of Equation 3 is given in Appendix A. (This expression for $\hat{h}(f)$ can also be derived using a minimum variance method, see Appendix A of [8] for details.)

\subsection{Computing the weighting functions}

In order to apply the formula given in equation 3 in the time-domain, we must compute the optimal combination for each frequency in the detection band, and convert this array of 'weights' into time-domain filters.

The variance terms, $\sigma_{\mathrm{PP}}$ and $\sigma_{\mathrm{QQ}}$, in equation 3 can be estimated by looking at the noise components of the two calibrated signals at each frequency; in other words, by considering the noise floor of the power spectral density (PSD) of each signal. (The variance of a particular signal at a given frequency is proportional to the mean value of the PSD at that frequency.) How good an estimate this is depends on the observation time and stationarity of the signal when making the PSD. If the noise is stationary, then the estimate of the PSD, and hence of the variance, can be improved by taking more and more averages. If the noise is non-stationary, then the PSD needs to be constructed over the appropriate time-scale. The terms $\sigma_{\mathrm{PQ}}$ and $\sigma_{\mathrm{QP}}$ can be estimated for each frequency by considering the noise floor of the cross-power spectral density of $h_{\mathrm{P}}$ and $h_{\mathrm{Q}}$; the same considerations of stationarity and observation time apply. 


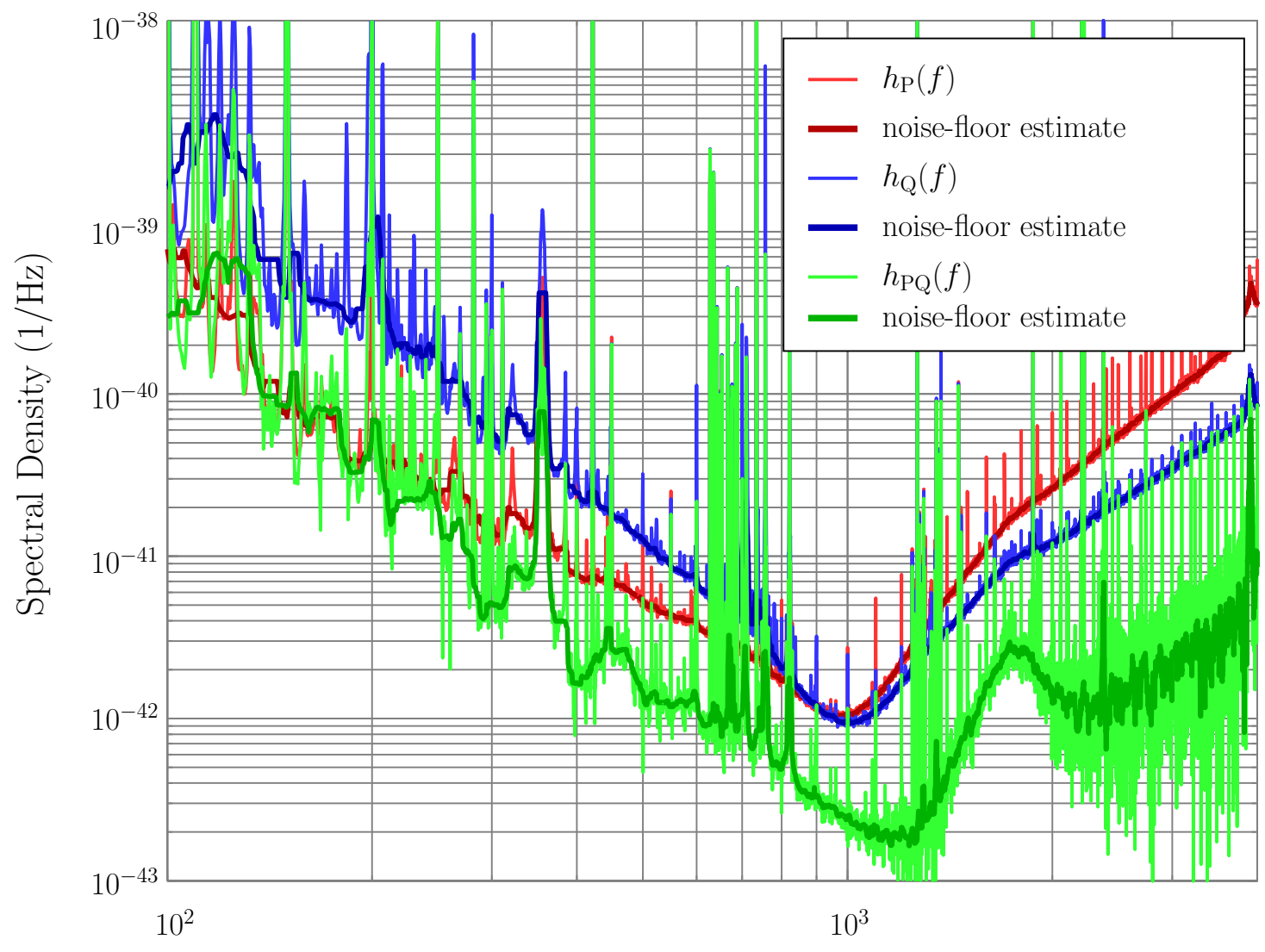

Frequency $(\mathrm{Hz})$

Figure 1. Power- and cross-spectral densities of the two calibrated outputs of GEO 600. Noise-floor estimates constructed from a running median estimator are also shown.

Figure 1 shows typical power spectral density estimates of $h_{\mathrm{P}}$ and $h_{\mathrm{Q}}$, together with an estimate of the cross-power spectral density (CSD); a noise floor estimate for each is also shown. The phase components of the CSD are shown in Figure 2. The noise floor estimates are constructed by using a running median estimator. For a particular frequency bin, $f$, the values in the surrounding $N$ bins are sorted in ascending order and the top $h_{\text {cut }} \%$ are discarded in order to discard outliers (from lines, for example). The median of the remaining values is taken as an estimate of the noise floor at frequency $f$. For the noise floor estimates shown in Figure $1, N=32$ bins, and $h_{\text {cut }}=0.9$.

Equation 3 can be re-written such that we have two complex frequency-dependent weighting factors, $A(f)$ and $B(f)$, for $h_{\mathrm{P}}$ and $h_{\mathrm{Q}}$ :

$$
\hat{h}(f)=A(f) h_{\mathrm{P}}(f)+B(f) h_{\mathrm{Q}}(f),
$$




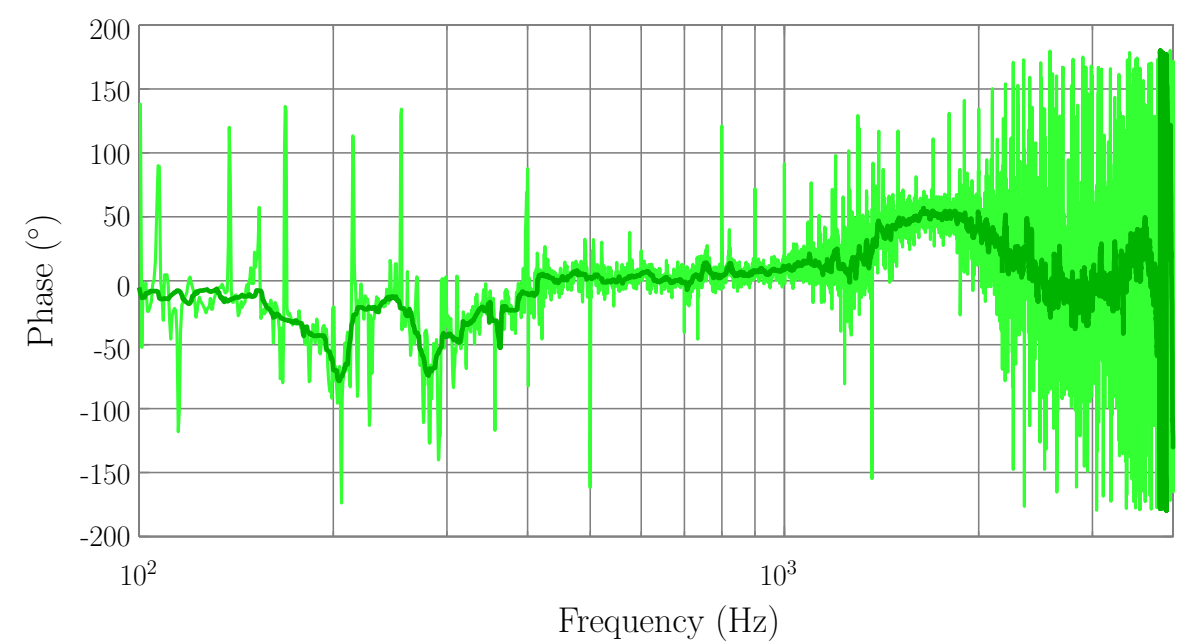

Figure 2. The phase components of the cross-spectral density of $h_{\mathrm{P}}(t)$ and $h_{\mathrm{Q}}(t) . A$ 'noise-floor estimate' is also shown.

where,

$$
\begin{aligned}
& A(f)=\frac{\sigma_{\mathrm{QQ}}(f)-\sigma_{\mathrm{PQ}}(f)}{\sigma_{\mathrm{PP}}(f)+\sigma_{\mathrm{QQ}}(f)-\left[\sigma_{\mathrm{PQ}}(f)+\sigma_{\mathrm{QP}}(f)\right]}, \\
& B(f)=\frac{\sigma_{\mathrm{PP}}(f)-\sigma_{\mathrm{QP}}(f)}{\sigma_{\mathrm{PP}}(f)+\sigma_{\mathrm{QQ}}(f)-\left[\sigma_{\mathrm{PQ}}(f)+\sigma_{\mathrm{QP}}(f)\right]} .
\end{aligned}
$$

Figure 3 shows an example of the magnitude and phase of the weighting functions for the data used in Figures 1 and 2.

\subsection{Designing the filters}

The weighting functions that were computed in the previous section are, in general, complex. In order to make time-domain filter representations of these two weighting functions, we need a way to compute time-domain filters that have arbitrary magnitude and phase responses. One such method is to consider the magnitude and phase responses separately. We can write $A(f)$ and $B(f)$ in terms of their magnitude and phase parts, such that

$$
\begin{aligned}
& A(f)=a(f) e^{i \phi_{a}(f)}, \\
& B(f)=b(f) e^{i \phi_{b}(f)} .
\end{aligned}
$$

Now we construct 4 filters, $\mathcal{M}_{\mathrm{P}}, \mathcal{P}_{\mathrm{P}}, \mathcal{M}_{\mathrm{Q}}$, and $\mathcal{P}_{\mathrm{Q}}$ which have the frequency responses, $a(f), e^{i \phi_{a}(f)}, b(f)$, and $e^{i \phi_{b}(f)}$ respectively. 


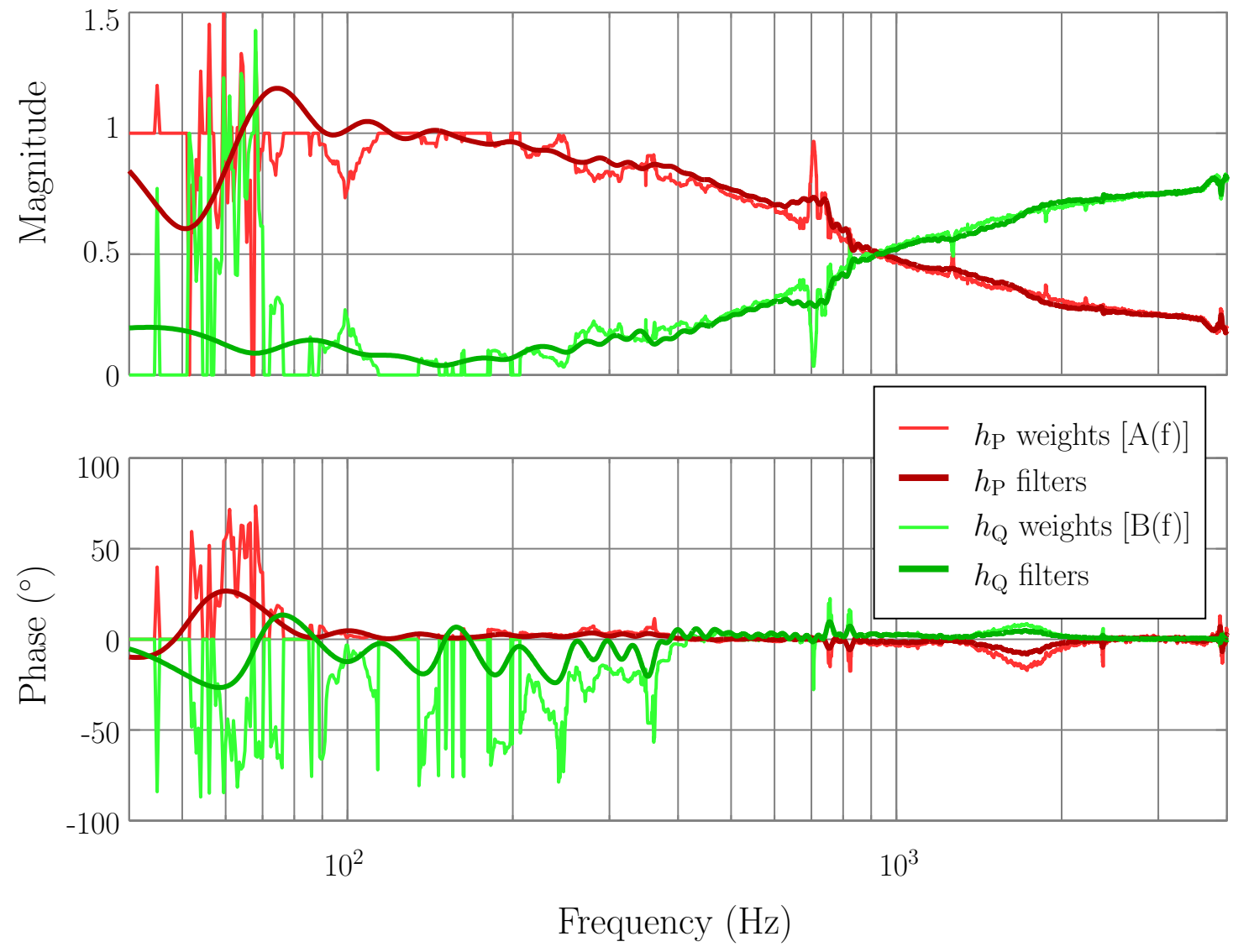

Figure 3. The optimal weighting functions for combining signals $h_{\mathrm{P}}(f)$ and $h_{\mathrm{Q}}(f)$ which have the power- and cross-spectral densities shown in figure 1. Also shown are the overall magnitude and phase responses of the filters pairs designed using the method described in the text.

Construction of the time-domain filters, $\mathcal{M}_{\mathrm{P}}$ and $\mathcal{M}_{\mathrm{Q}}$, is done by assuming that the response we seek $(a(f)$ and $b(f))$ are the Fourier transforms of the impulse responses of the two linear-phase time-domain filters [9, 10, 11]. For this to be the case, we need to generate the appropriate phase delay for the two filters. This is just a frequency dependent linear phase shift defined by the filter order we require. If we want filters of order $N_{\text {taps }}$, then we construct a grid of $N_{\mathrm{G}}$ equally spaced frequency points that run from 0 to the Nyquist rate and estimate the magnitude and phase at each point. The magnitude estimates come directly from $a(f)$ and $b(f)$ (interpolated or averaged as necessary), and the phase components are just linear with frequency starting from 0 phase shift, such that

$$
\tilde{\mathcal{M}}_{\mathrm{P}}(f)=a(j) \exp \left[\frac{-\left(N_{\text {taps }}-1\right) i \pi j}{2\left(N_{\mathrm{G}}-1\right)}\right]
$$




$$
\tilde{\mathcal{M}}_{\mathrm{Q}}(f)=b(j) \exp \left[\frac{-\left(N_{\text {taps }}-1\right) i \pi j}{2\left(N_{\mathrm{G}}-1\right)}\right],
$$

where $\tilde{\mathcal{M}}_{\mathrm{P}}(f)$ denotes the response of the filter $\mathcal{M}_{\mathrm{P}}$ (the single-sided Fourier transform of the impulse response), $i=\sqrt{-1}$, and $j \in\left[0: N_{\mathrm{G}}\right)$.

We can then form the two-sided Fourier transform of the impulse response of the filter by concatenating the vector $\tilde{\mathcal{M}}_{\mathrm{P}}(f)$ with the reverse sequence of the conjugate of $\tilde{\mathcal{M}}_{\mathrm{P}}(f)$; the same is done for $\tilde{\mathcal{M}}_{\mathrm{Q}}(f)$. Thus we have a frequency series that runs from $-f_{\mathrm{s}} / 2$ to $+f_{\mathrm{s}} / 2$. This is what we would get from the Fourier Transform of a real data series. If we take the inverse transform, and apply a window function, we get the filter coefficients we seek for $\mathcal{M}_{\mathrm{P}}$ and $\mathcal{M}_{\mathrm{Q}}$. The time shifts introduced when applying these filters can simply be removed by appropriate buffering of the filtered signals.

The filters representing the phase components of the weighting functions are constructed as all-pass filters. Here the magnitude response of the filter is designed to be unity for a significant part of the pass-band. The phase response is an approximation to that phase response we seek. These filters can be designed by minimising the difference between the desired response, $e^{i \phi_{a}(f)}$, and the response of the filter, $\tilde{\mathcal{P}}_{\mathrm{P}}(f)$ using, for example, a non-linear least-squares routine. The error-function that was minimised in this application was

$$
\epsilon=\Sigma_{k}\left|\tilde{\mathcal{P}}_{\mathrm{P}}\left(f_{k}\right)-e^{i \phi_{a}\left(f_{k}\right)}\right|^{2}
$$

An example of the combined magnitude and phase response of $\mathcal{M}_{\mathrm{P}}$ with $\mathcal{P}_{\mathrm{P}}$, and $\mathcal{M}_{\mathrm{Q}}$ with $\mathcal{P}_{\mathrm{Q}}$ is shown in Figure 3. Also shown are the original weighting functions. (The linear-phase response of $\mathcal{M}_{\mathrm{P}}$ and $\mathcal{M}_{\mathrm{Q}}$ is omitted for clarity). The examples shown are 300 tap filters for the magnitude parts, and 512 tap filters for the phase parts.

\subsection{Results}

Having constructed the time-domain filters, we can easily compute the optimal $h(t)$ signal in the time-domain by

$$
h(t)=\tilde{\mathcal{M}}_{\mathrm{P}}\left\{h_{\mathrm{P}}(t)\right\}+\tilde{\mathcal{P}}_{\mathrm{P}}\left\{h_{\mathrm{P}}(t)\right\}+\tilde{\mathcal{M}}_{\mathrm{Q}}\left\{h_{\mathrm{Q}}(t)\right\}+\tilde{\mathcal{P}}_{\mathrm{Q}}\left\{h_{\mathrm{Q}}(t)\right\}
$$

The result of applying this to real GEO 600data is shown in Figure 4. Here we see three amplitude spectral densities constructed from $h_{\mathrm{P}}(t), h_{\mathrm{Q}}(t)$, and $h(t)$. 


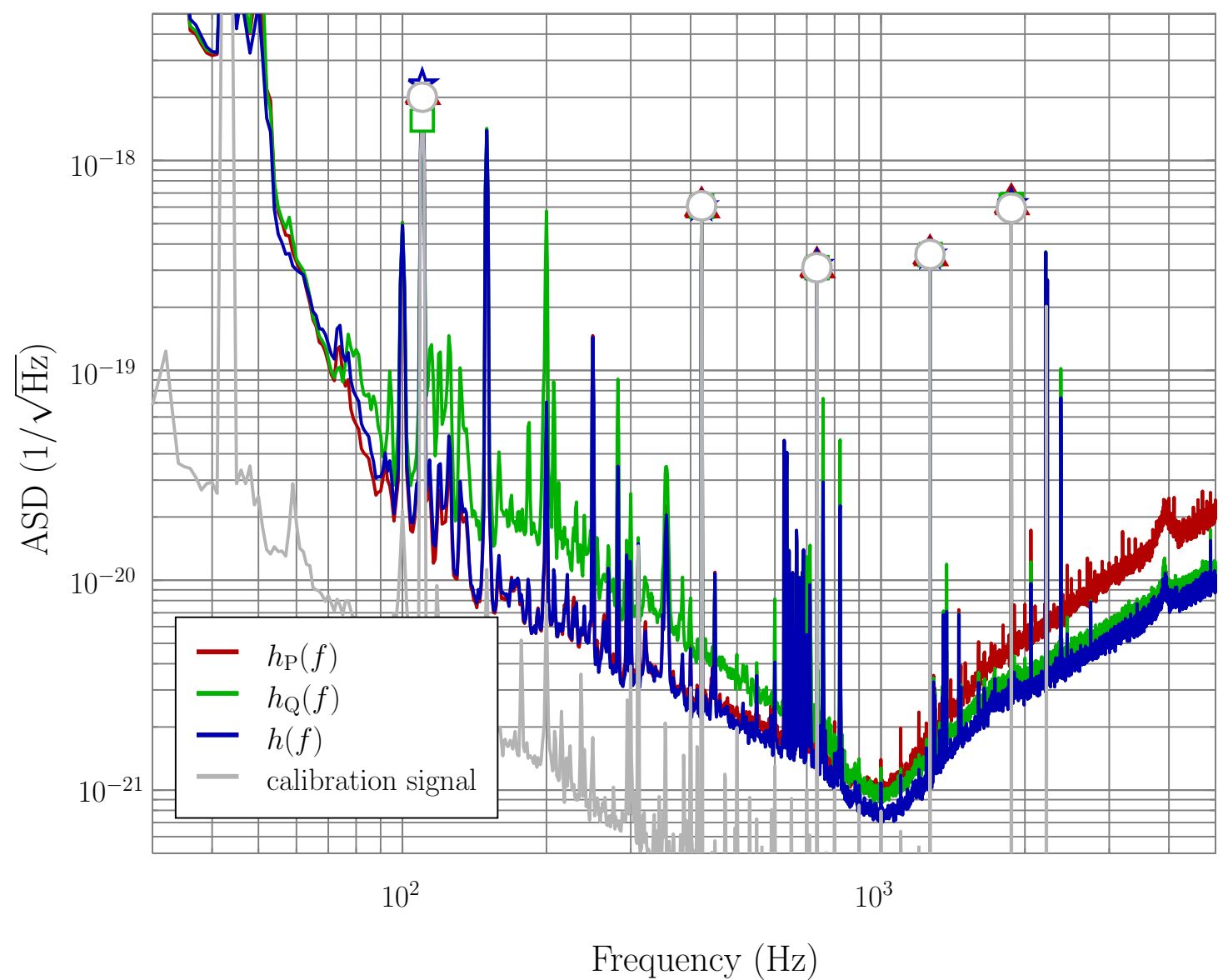

Figure 4. Snap-shot amplitude spectral densities (ASD) of the two calibrated outputs of GEO 600, $h_{\mathrm{P}}(t), h_{\mathrm{Q}}(t)$, and of the optimally combined signal, $h(t)$. Injected calibration lines are highlighted with filled markers. An ASD of the strain induced by the injected calibration signal is also shown with the peaks marked by the grey circles.

An amplitude spectral density of the injected calibration signal calibrated to strain is also shown. The calibration peaks used for determining the detector response are indicated with filled markers. In order to confirm that any gravitational wave signal in $h(t)$ is not corrupted during the combining process, we can look at a relative comparison of the calibrated and combined strain signals to the induced strain signal. By computing the magnitude and phase of the calibration peaks in all signals, we can form the magnitude ratio and phase difference between the induced strain, and the three computed strain signals. Figure 5 shows the results of this calculation for the data stretch shown in Figure 4. We can see that, at least at these spot-frequencies, the combined $h(t)$ preserves the signal present in the underlying $h_{\mathrm{P}}(t)$ and $h_{\mathrm{Q}}(t)$ to a good degree. The phase-lag seen at low frequencies in this comparison is the result of two high-pass 
filters; one applied in the calibration routine to restrict the dynamic range of the signals, and one applied in the spectral analysis to reduce the effect of spectral leakage from the window function. We can also see from Figure 5 that the combination of the two calibrated data streams is poorer at low frequencies compared to high frequencies. There are two possible reasons for this: the calibration of the individual strain signals, $h_{\mathrm{P}}(t)$ and $h_{\mathrm{Q}}(t)$, is less good at these frequencies due to effect of the Michelson length-control servo (which has unity gain around $100 \mathrm{~Hz}$ ) on the calibration model; a series of strong lines and features around $100 \mathrm{~Hz}$ makes it more difficult to accurately estimate the noise floor and hence to create suitable time-domain filters.
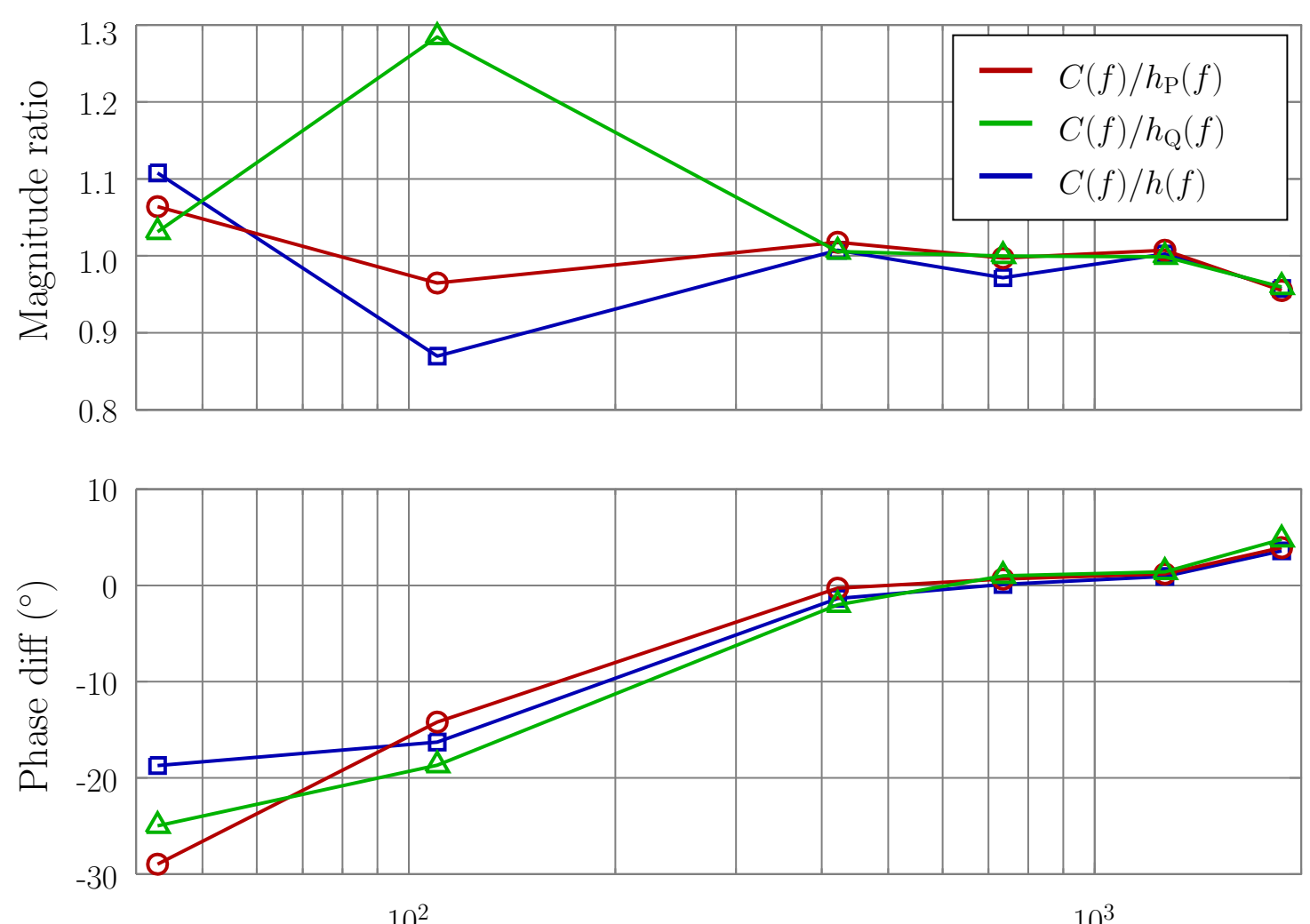

$10^{2}$

$10^{3}$

Frequency $(\mathrm{Hz})$

Figure 5. The magnitude ratio and phase difference of the strain induced by injecting calibration lines to the detected strain as measured in the two calibrated output signals, as well as the combined strain signal. The comparison is made at each calibration line frequency. 


\section{Summary and future work}

The on-line calibration scheme used at GEO 600 was extended to include an optimal time-domain combination of the two calibrated output signals. The result is a single $h(t)$ signal that is at least as sensitive as either of the two calibrated output signals at all frequencies in the detection band, and is a significant improvement at some frequencies. One further step is to allow for time-variation in the optimal combination filters. This means determining the filters on-line periodically, and then smoothly moving from using one set of filters to the next. This is in principle straightforward, with the complexity lying only in the implementation since we wish to maintain the real-time, low-latency nature of the current system.

\section{Acknowledgements}

The authors would like to thank Albert Lazzerini and Joe Romano for their help and discussions regarding the methods presented in [8].

\section{Appendix A. Maximum likelihood estimator for $h(t)$}

We are given two pieces of data, $h_{\mathrm{p}}$ and $h_{\mathrm{q}}$, which are generally complex and are related to a complex parameter $h$ by

$$
\begin{aligned}
& h_{\mathrm{p}}=h+N_{\mathrm{p}} \\
& h_{\mathrm{q}}=h+N_{\mathrm{q}} .
\end{aligned}
$$

We write this in vector form as

$$
\mathbf{d}=\mathbf{h}+\mathbf{N}
$$

The noises are complex and correlated with a (Hermitian) covariance matrix

$$
\mathbf{C}=\left(\begin{array}{cc}
\sigma_{\mathrm{pp}} & \sigma_{\mathrm{pq}} \\
\sigma_{\mathrm{qp}} & \sigma_{\mathrm{qq}}
\end{array}\right)
$$

where

$$
\sigma_{i j}=\left\langle N_{i} N_{j}^{*}\right\rangle
$$


and ${ }^{*}$ denotes complex conjugate. The determinant of this matrix is

$$
\operatorname{det} \mathrm{C}=\sigma_{\mathrm{pp}} \sigma_{\mathrm{qq}}-\sigma_{\mathrm{qp}} \sigma_{\mathrm{pq}}=\sigma_{\mathrm{pp}} \sigma_{\mathrm{qq}}-\left|\sigma_{\mathrm{pq}}\right|^{2}
$$

and its inverse is

$$
\mathrm{C}^{-1}=\frac{1}{\operatorname{det} \mathrm{C}}\left(\begin{array}{cc}
\sigma_{\mathrm{qq}} & -\sigma_{\mathrm{pq}} \\
-\sigma_{\mathrm{qp}} & \sigma_{\mathrm{pp}}
\end{array}\right) .
$$

The likelihood of $h$ is the probability of the data given $h$, taken to be a complex bivariate gaussian, i.e.,

$$
p\left(h_{\mathrm{p}}, h_{\mathrm{q}} \mid h\right)=\frac{1}{\pi^{2} \operatorname{det} \mathrm{C}} \exp \left[-(\mathbf{d}-\mathbf{h})^{\mathrm{H}} \mathbf{C}^{-1}(\mathbf{d}-\mathbf{h})\right],
$$

with $\mathrm{x}^{\mathrm{H}}$ the conjugate transpose of $\mathbf{x}$. The maximum likelihood estimator for $h$ is the value that maximises this probability, or alternatively minimises

$$
\begin{aligned}
Q & =(\mathbf{d}-\mathbf{h})^{\mathrm{H}} \mathbf{C}^{-1}(\mathbf{d}-\mathbf{h}) \\
& =\mathbf{d}^{\mathrm{H}} \mathbf{C}^{-1} \mathbf{d}+\mathbf{h}^{\mathrm{H}} \mathbf{C}^{-1} \mathbf{h}-2 \Re\left\{\mathbf{h}^{\mathrm{H}} \mathbf{C}^{-1} \mathbf{d}\right\}
\end{aligned}
$$

with respect to the real and imaginary components of $h$. Our constraints are therefore

$$
\frac{\partial Q}{\partial \Re\{h\}}=0 ; \quad \frac{\partial Q}{\partial \Im\{h\}}=0 .
$$

Multiplying out individual terms we get

$$
\mathbf{h}^{\mathrm{H}} \mathbf{C}^{-1} \mathbf{h}=\frac{h h^{*}}{\operatorname{det} \mathrm{C}}\left(\sigma_{\mathrm{pp}}+\sigma_{\mathrm{qq}}-\sigma_{\mathrm{pq}}-\sigma_{\mathrm{qp}}\right)
$$

and

$$
\mathbf{h}^{\mathrm{H}} \mathbf{C}^{-1} \mathbf{d}=\frac{h^{*}}{\operatorname{det} \mathrm{C}}\left[h_{\mathrm{p}}\left(\sigma_{\mathrm{qq}}-\sigma_{\mathrm{pq}}\right)+h_{\mathrm{q}}\left(\sigma_{\mathrm{pp}}-\sigma_{\mathrm{qp}}\right)\right],
$$

so the maximum likelihood estimator of $h$ is

$$
\hat{h}_{\mathrm{ML}}=\frac{h_{\mathrm{p}}\left(\sigma_{\mathrm{qq}}-\sigma_{\mathrm{pq}}\right)+h_{\mathrm{q}}\left(\sigma_{\mathrm{pp}}-\sigma_{\mathrm{qp}}\right)}{\left(\sigma_{\mathrm{pp}}+\sigma_{\mathrm{qq}}\right)-\left(\sigma_{\mathrm{pq}}+\sigma_{\mathrm{qp}}\right)} .
$$

Note the denominator of this is purely real. 
[1] Commissioning, characterization, and operation of the dual-recycled GEO $600 \mathrm{~J}$ R Smith et al, Class. Quantum Grav. 21 (7 March 2004) S1737-S1745.

[2] Dual recycling for GEO $600 \mathrm{G}$ Heinzel et al, Class. Quantum Grav. 19 No 7, 7th April 2002.

[3] Dual recycling for GEO 600, H Grote, A Freise, M Malec, G Heinzel, B Willke, H Lück, K A Strain, J Hough and K Danzmann, Class. Quantum Grav. 21 No 5 (7 March 2004) S473-S480.

[4] Calibration of the power-recycled gravitational wave detector, GEO600, M Hewitson, H Grote, G Heinzel, K A Strain, H Ward, U Weiland, Rev. Sci. Instrum. 74, 4184 (2003).

[5] Calibration of GEO 600 for the S1 science run,M Hewitson, H Grote, G Heinzel, K A Strain, H Ward and U Weiland, Class. Quantum Grav. 20 No 17 (7 September 2003) S885-S893.

[6] Principles of calibrating the dual-recycled GEO 600, M Hewitson, G Heinzel, J R Smith, K A Strain, and H Ward, Rev. Sci. Instrum. 75, 4702 (2004).

[7] Calibration of the dual-recycled GEO 600 detector for the S3 science run, M Hewitson, S Babak, R Balasubramanian, K Danzmann, H Grote, G Heinzel, J Hough, H Lück, M A Papa, J R Smith, K A Strain, H Ward, B Willke and G Woan, Class. Quantum Grav. 21 No 20 (21 October 2004) S1711-S1722.

[8] Optiaml combination of signals from colocated gravitational wave interferometers for use in searches for a stochastic background, A Lazzerini et al, Phys. Rev. D, 70, 2001.

[9] Digital Signal Processing A Computer Based Approach, First Edition, S K Mitra, McGraw-Hill, New York, 1998, pp. 462-468.

[10] Digital Filters and Signal Processing, Third Edition, L B Jackson, Kluwer Academic Publishers, Boston, 1996, pp. 301-307.

[11] Discrete-time Signal Processing, Second Edition, A V Oppenheim, R W Shafer, Prentice Hall Signal Processing Series, 1999. 